\section{STREITPUNKT KOHLENDIOXID}

Ein ambitioniertes Ziel, denn immerhin gilt: Andere Länder, andere Sitten. Dennoch haben sich auf Einladung der Economic Commission for Europe (UN-ECE) ausgewählte Vertreter nationaler Normierungsinstitute zusammengefunden, um die Fahrdaten der fünf Regionen EU mit der Schweiz, USA, Indien, Südkorea und Japan in einem Standard, dem World-wide Harmonized Ligh Duty Driving Test Cycle (WLTC), zu bündeln. Allerdings ist China, der mittlerweile größte Automobilmarkt der Welt, nicht aktiv im illustren Kreis vertreten. Das Land hat seine eigenen Vorgaben, wird sich aber am WLTP orientieren.

So unterschiedlich das Fahrverhalten in den jeweiligen Ländern auch ist: Allen gemeinsam ist, dass das Kohlendioxid als limitierender Faktor für den Mehrverbrauch künftiger Neufahrzeuge beibehalten wird. Ein Blick auf die $\mathrm{CO}_{2}$-Grenzwerte der großen Automobilmärkte zeigt, dass die EU mit Abstand die strengsten Vorgaben beschlossen hat. Wenn ab 2020 in Europa nur noch $95 \mathrm{~g} \mathrm{CO}_{2} / \mathrm{km}$ erlaubt sind, haben die USA noch fünf weitere Jahre Zeit, diesen Wert umzusetzen. Zwar verzichtet die EU auf einheitliche Grenzwerte für alle OEMs und gesteht ihnen herstellerspezifische Grenzwerte zu, die sich nach einem komplizierten Schlüssel ergeben, bei dem unter anderem das Fahrzeuggewicht, Zulassungszahlen und ein Gewichtungsfaktor berücksichtigt werden.

Doch Verbrauch und Emissionen jedes Neuwagens werden im offiziellen Messverfahren ermittelt, und jede Grenzwertüberschreitung wird bestraft. Die Höhe hängt von dem Ausmaß der Überschreitung und der Zahl der verkauften Neuwagen $a b$. Berechnungen des Instituts der deutschen Wirtschaft Köln zufolge kann dann schnell eine Strafe in Milliardenhöhe folgen [5].

Vor allem diese unmittelbaren Folgen empfinden insbesondere die deutschen OEMs als schmerzhaftes Limit. Für sie ist es deshalb „ganz entscheidend, wie die Überleitungsformeln vom NEFZ, der ja die bekannte gesetzlich verabschiedete $\mathrm{CO}_{2}$-Flottenemission auf $95 \mathrm{~g} / \mathrm{km} \mathrm{ab}$ 2020 begrenzt, ermittelt werden und final zur Verabschiedung kommen“, erklärt Gutzmer.

\section{BEIM WLTP BLOSS KEINE REGIONALEN BESONDERHEITEN}

Lässt sich eine Einigung erzielen, dann wäre dies eine gute Nachricht. Aus gutem Grund. Bisher werden etwa in den USA oder Japan Fahrzyklen angewandt, die teilweise erheblich vom NEFZ abweichen. Das hat

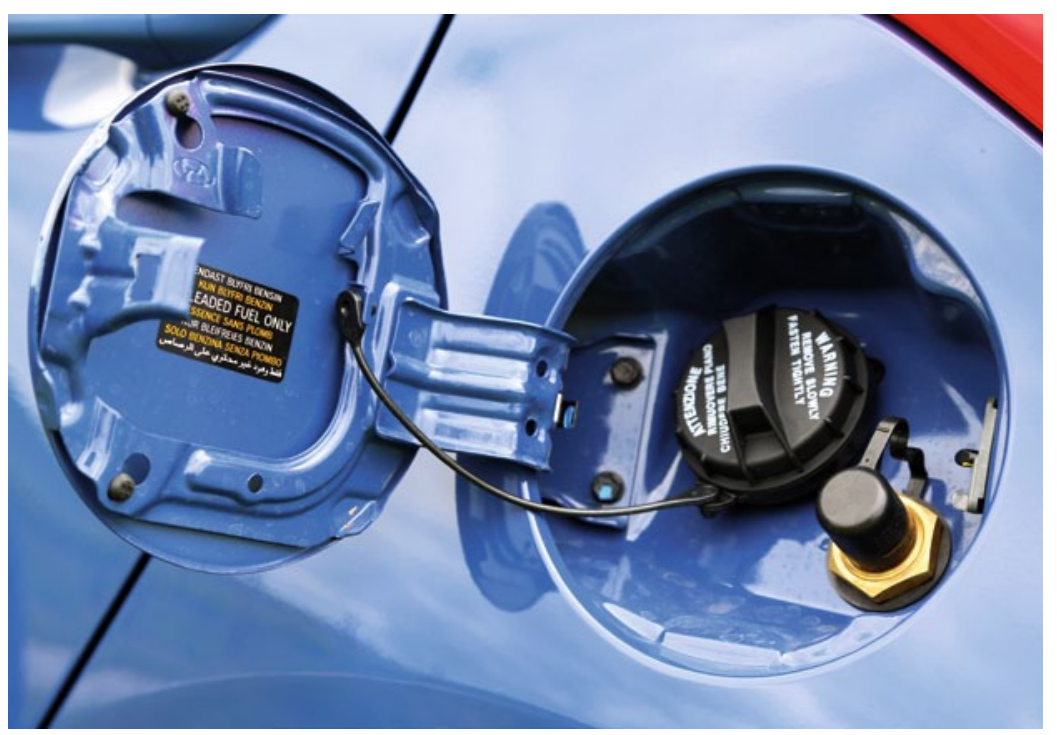

Ob Benzin oder Flüssiggas: der Verbraucher fordert sein Recht, die angegebenen Verbrauchswerte auch erreichen zu können (Bild @ Andreas Burkert)
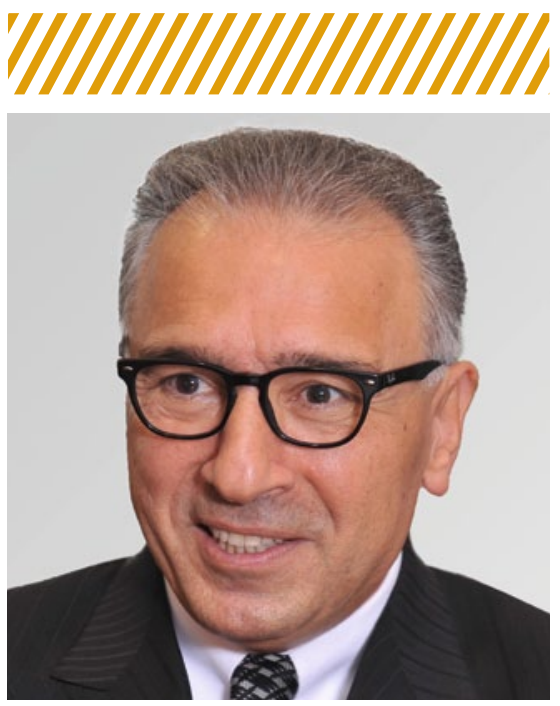

PROFESSOR DR.-ING. FERIT KÜÇÜKAY

Direktor am Institut für Fahrzeugtechnik der TU Braunschweig

\section{FRAGEN AN ...}

\section{ATZ _ Wie wirkt sich der WLTP auf die} Systemauslegung eines Antriebsstrangs aus? KÜçÜKAY_Auch die Rückgewinnung der kinetischen Energie (Rekuperation) ist im WLTP nicht effektiver als im NEFZ. Daher wird der Fokus der Entwicklung noch stärker auf Maßnahmen liegen, die eine Verringerung der ,irreversiblen“ Fahrwiderstände zur Folge haben. Insbesondere Verbesserungen des Luftwiderstands wirken sich im WLTP stärker aus als im NEFZ. Darüber hinaus werden die geänderten Prüfbedingungen im WLTP auch die Getriebeauslegung beeinflussen.

\section{Sie bemängeln einige offene Punkte} und Ungereimtheiten im WLTP. Hat sich diesbezüglich mittlerweile etwas zum Positiven verändert?

Nein. Eine Herausforderung bei der Umstellung des NEFZ auf den WLTP bleibt die Adaption der Emissions- und Flottengrenzwerte auf den neuen Zyklus. Da das Geschwindigkeitsprofil und die Zyklusrandbedingungen des WLTP mit dem NEFZ nicht vergleichbar sind, können auch die gesetzlich festgelegten Grenzwerte für die Schadstoff- und $\mathrm{CO}_{2}$ Emissionen nicht einfach übertragen werden. Die aktuell geplante Umrechnung über mathematische Modelle wird mit hoher Wahrscheinlichkeit nicht präzise genug sein, um von allen OEMs akzeptiert zu werden.

INTERVIEW: Andreas Burkert 